\title{
ON THE ASYMPTOTIC REPRESENTATION OF THE SOLUTIONS TO THE FOURTH GENERAL PAINLEVÉ EQUATION
}

\author{
YOUMIN LU
}

Received 5 June 2002

\begin{abstract}
There have been many results on the asymptotics of the Painlevé transcendents in recent years, but the asymptotics of the fourth Painlevé transcendent has not been studied much. In this note, we study the general fourth Painlevé equation and develop an asymptotic representation of a group of its solutions as the independent variable approaches infinity along a straight line.
\end{abstract}

2000 Mathematics Subject Classification: 34E99.

1. Introduction. Asymptotic behaviour of the second and the third Painlevé transcendents has been much studied $[1,3,4,7]$. But there are very few results about the fourth Painlevé equation

$$
\frac{d^{2} \eta}{d \xi^{2}}=\frac{1}{2 \eta}\left(\frac{d \eta}{d \xi}\right)^{2}+\frac{3}{2} \eta^{3}+4 \xi \eta^{2}+\left(\xi^{2}-\alpha\right) \eta+\frac{\beta}{\eta}
$$

where $\alpha$ and $\beta$ are parameters. In [2], Clarkson and McLeod studied the special case of (1.1) for the parameter $\beta=0$ and obtained the complete asymptotic representation of its solution with $\eta(\infty)=0$. In [6], we studied the general case of (1.1) and proved the following theorem.

THEOREM 1.1. Under the assumption that $i \alpha \geq 0$ and $\beta>0$, (1.1) has a solution $\eta(\xi)$ with the following asymptotics:

(i) as $\xi \rightarrow e^{\pi i / 4} \infty$,

$$
\begin{gathered}
\eta(\xi)=\frac{i r}{\xi} \pm \frac{i}{\xi}\left(\sqrt{r^{2}-\frac{\beta}{2}}+O\left(\xi^{-2}\right)\right) \cos \phi, \\
\eta^{\prime}(\xi)= \pm 2\left(\sqrt{r^{2}-\frac{\beta}{2}}+O\left(\xi^{-2}\right)\right) \sin \phi,
\end{gathered}
$$

where $\phi=i \xi^{2}-(1 / 2)(3 r+i \alpha) \log \left(-i \xi^{2}\right)+\theta_{0}+O\left(\xi^{-2}\right)$, and $r>0$ and $\theta_{0}$ are real parameters satisfying $r^{2}-\beta / 2>0$;

(ii) as $\xi \rightarrow e^{\pi i / 4} 0$,

$$
\eta(\xi)=e^{\pi i / 4}\left(a+b e^{-\pi i / 4} \xi+O\left(\xi^{2}\right)\right),
$$

where $a$ and $b$ are real parameters with $a>0$. 
Furthermore, any solution of (1.1) of the form $\eta(\xi)=e^{\pi i / 4} W(\xi)$ with $W(\xi) \geq 0$ has the above asymptotics.

A natural question following this result is how this solution behaves when $\xi$ goes to infinity in the opposite direction of the line. As we know, through the transformations

$$
\eta(\xi)=e^{-\pi i / 4} y, \quad \xi=e^{-\pi i / 4} x
$$

(1.1) is related to the equation

$$
\frac{d^{2} y}{d x^{2}}=\frac{1}{2 y}\left(\frac{d y}{d x}\right)^{2}-\frac{3}{2} y^{3}-4 x y^{2}-2\left(x^{2}+\hat{\alpha}\right) y+\frac{\beta}{y}
$$

where $\alpha=i \hat{\alpha}$. Applying the transformation

$$
y(x)=-x w^{2}(t), \quad t=-x^{2},
$$

we can obtain the equation

$$
w^{\prime \prime}+t^{-1} w^{\prime}=\frac{1}{16} t^{-2} w-\frac{3}{16} w^{5}+\frac{1}{2} w^{3}-\frac{1}{4} w+\frac{\hat{\alpha}}{4} t^{-1} w+\frac{\beta}{8 t^{2} w^{3}}
$$

It is easy to prove that $w, w^{\prime}$, and $1 / t^{2} w^{2}$ are all bounded as $t \rightarrow+\infty$ and when $\hat{\alpha} \leq 0$ and $\beta \geq 0$. Inspecting (1.7) carefully, we can conclude that there are possibly four cases for the behaviour of $w$ as $t \rightarrow+\infty$ :

(1) $w(t) \rightarrow 0$ as $t \rightarrow+\infty$;

(2) $w(t) \rightarrow \sqrt{2}$ as $t \rightarrow+\infty$;

(3) $w(t) \rightarrow \sqrt{2 / 3}$ as $t \rightarrow+\infty$;

(4) $w$ does not have a limit as $t \rightarrow+\infty$.

Case (1) has been studied and eliminated in [5]. In this note, we pay our attention to case (2) and develop the asymptotic representation of $\eta$ corresponding to this case as $\xi \rightarrow e^{5 \pi i / 4}(+\infty)$.

2. Development of the asymptotic representation. From now on, we assume that $\hat{\alpha} \leq 0, \beta \geq 0$, and $w(t) \rightarrow \sqrt{2}$ as $t \rightarrow+\infty$. We first prove the following theorem.

THEOREM 2.1. The function $W$ is bounded as $t \rightarrow+\infty$ if $w=\sqrt{2}+t^{-1 / 2} W$.

Proof. Applying the transformation $w=\sqrt{2}+t^{-1 / 2} W$ to (1.7), we obtain

$$
\begin{aligned}
W^{\prime \prime}= & \frac{\sqrt{2}}{16} t^{-3 / 2}-\frac{3}{16} t^{-2} W-W-\frac{9 \sqrt{2}}{4} t^{-1 / 2} W^{2}-\frac{13}{4} t^{-1} W^{3} \\
& -\frac{15 \sqrt{2}}{16} t^{-3 / 2} W^{4}-\frac{3}{16} t^{-2} W^{5}+\frac{\hat{\alpha} \sqrt{2}}{4} t^{-1 / 2}+\frac{\hat{\alpha}}{4} t^{-1} W+\frac{\beta t^{-3 / 2}}{8 w^{3}} .
\end{aligned}
$$


Multiplying both sides of (2.1) by $2 W^{\prime}$ and integrating it, we have

$$
\begin{aligned}
W^{\prime 2}+W^{2}= & \frac{\sqrt{2}}{8} t^{-3 / 2} W+\frac{3 \sqrt{2}}{16} \int_{t_{0}}^{t} t^{-5 / 2} W d t-\frac{3}{16} t^{-2} W^{2}-\frac{3}{8} \int_{t_{0}}^{t} t^{-3} W^{2} d t \\
& -\frac{3 \sqrt{2}}{2} t^{-1 / 2} W^{3}-\frac{3 \sqrt{2}}{4} \int_{t_{0}}^{t} t^{-3 / 2} W^{3} d t-\frac{13}{8} t^{-1} W^{4}-\frac{13}{8} \int_{t_{0}}^{t} t^{-2} W^{4} d t \\
& -\frac{3 \sqrt{2}}{8} t^{-3 / 2} W^{5}-\frac{9 \sqrt{2}}{16} \int_{t_{0}}^{t} t^{-5 / 2} W^{5} d t-\frac{1}{16} t^{-2} W^{6}-\frac{1}{8} \int_{t_{0}}^{t} t^{-3} W^{6} d t \\
& +\frac{\hat{\alpha} \sqrt{2}}{2} t^{-1 / 2} W+\frac{\hat{\alpha} \sqrt{2}}{4} \int_{t_{0}}^{t} t^{-3 / 2} W d t+\frac{\hat{\alpha}}{4} t^{-1} W^{2}+\frac{\hat{\alpha}}{4} \int_{t_{0}}^{t} t^{-2} W^{2} d t \\
& -\frac{\beta}{8 t w^{2}}-\frac{\beta \sqrt{2}}{8} \int_{t_{0}}^{t} \frac{d t}{t^{2} w^{3}}+C_{1} .
\end{aligned}
$$

Clearly, $W^{\prime 2}(t)=o(t)$, and therefore $C_{1}=o\left(t_{0}\right)$. Because $t^{-1 / 2} W=o(1)$ as $t \rightarrow+\infty, \int_{t_{0}}^{t} t^{-5 / 2} W d t$ is bounded and the following inequalities are true for large $t_{0}$ and $t \geq t_{0}$ :

$$
\begin{gathered}
\frac{13}{8} \int_{t_{0}}^{t} t^{-2} W^{4} d t+\frac{9 \sqrt{2}}{16} \int_{t_{0}}^{t} t^{-5 / 2} W^{5} d t \geq \frac{1}{2} \int_{t_{0}}^{t} t^{-2} W^{4} d t \\
W^{2}+\frac{3 \sqrt{2}}{2} t^{-1 / 2} W^{3}+\frac{3 \sqrt{2}}{8} t^{-3 / 2} W^{5} \geq \frac{1}{2} W^{2}, \\
\frac{3 \sqrt{2}}{32} \int_{t_{0}}^{t} t^{-5 / 2}(|W|-W) d t-\frac{\hat{\alpha} \sqrt{2}}{8} \int_{t_{0}}^{t} t^{-3 / 2}(|W|-W) d t \\
\leq-\frac{\hat{\alpha} \sqrt{2}}{4} \int_{t_{0}}^{t} t^{-3 / 2}(|W|-W) .
\end{gathered}
$$

Hence, there exists a constant $C_{2}$ and a large $t_{0}$ such that

$$
\begin{array}{r}
W^{\prime 2}+\frac{1}{2} W^{2}+\frac{3}{16} t^{-2} W^{2}+\frac{3}{8} \int_{t_{0}}^{t} t^{-3} W^{2} d t+\frac{3}{8} t^{-1} W^{4}+\frac{1}{2} \int_{t_{0}}^{t} t^{-2} W^{4} d t \\
+\frac{1}{16} t^{-2} W^{6}+\frac{1}{8} \int_{t_{0}}^{t} t^{-3} W^{6} d t-\frac{\widehat{\alpha}}{4} t^{-1} W^{2}-\frac{\widehat{\alpha}}{4} \int_{t_{0}}^{t} t^{-2} W^{2} d t \\
+\frac{3 \sqrt{2}}{32} \int_{t_{0}}^{t} t^{-3 / 2}(|W|+W)^{3} d t+\frac{\hat{\alpha} \sqrt{2}}{8} \int_{t_{0}}^{t} t^{-3 / 2}(|W|+W) d t \\
\leq \frac{3 \sqrt{2}}{32} \int_{t_{0}}^{t} t^{-3 / 2}(|W|-W)^{3} d t-\frac{\hat{\alpha} \sqrt{2}}{4} \int_{t_{0}}^{t} t^{-3 / 2}(|W|-W) d t+C_{2} .
\end{array}
$$

From (2.1), we can claim that $\lim _{t \rightarrow+\infty} W(t) \neq-\infty$. If $\int_{t_{0}}^{t} t^{-3 / 2} W^{3} d t \geq 0$ and $\int_{t_{0}}^{t} t^{-3 / 2} W d t \geq 0$, then every term, especially $W^{2}$, in inequality (2.4) is bounded. If $W$ is bounded below, the same argument can be applied to obtain that $W^{2}$ is 
bounded for large $t$. If $W$ is not bounded below, from inequality (2.4), we have

$$
\begin{aligned}
\frac{1}{8}(|W|-W)^{2} \leq \frac{1}{2} W^{2} \leq & \frac{3 \sqrt{2}}{32} \int_{t_{0}}^{t} t^{-3 / 2}(|W|-W)^{3} d t \\
& -\frac{\hat{\alpha} \sqrt{2}}{8} \int_{t_{0}}^{t} t^{-3 / 2}(|W|-W) d t+C_{2} .
\end{aligned}
$$

Since $(|W|-W)^{3}>-(2 \hat{\alpha} / 3)(|W|-W)$ when $(|W|-W)$ is larger than a certain constant, there exists a constant $C_{3}>0$ and $C_{3}=o\left(t_{0}\right)$ such that

$$
(|W|-W)^{2} \leq \frac{3 \sqrt{2}}{2} \int_{t_{0}}^{t} t^{-3 / 2}(|W|-W)^{3} d t+C_{3} .
$$

Now, let $I$ be the right-hand side of (2.6). Then,

$$
I^{\prime}=\frac{3 \sqrt{2}}{2} t^{-3 / 2}(|W|-W)^{3} \leq \frac{3 \sqrt{2}}{2} t^{-3 / 2} I^{3 / 2} .
$$

Integrating both sides of (2.7), we obtain $1 / I^{1 / 2} \geq 1 / C_{3}^{1 / 2}-3 \sqrt{2} / 2 t_{0}^{1 / 2}+$ $3 \sqrt{2} / 2 t^{1 / 2}>0$ for large $t_{0}$. Thus, $I$ is bounded, and we reach a contradiction. Hence, $W$ is bounded below and the theorem is proved.

THEOREM 2.2. As $\xi \rightarrow \infty$ along $\arg \xi=5 \pi / 4$,

$$
\begin{gathered}
\eta(\xi)=-2 \xi \pm\left(2 e^{\pi i / 4} \sqrt{2} d+O\left(\xi^{-1}\right)\right) \cos \phi, \\
\eta^{\prime}(\xi)=-2 \mp 4 e^{3 \pi i / 4} \sqrt{2} d \xi \sin \phi+O\left(\xi^{-1}\right),
\end{gathered}
$$

where $\phi=-i \xi^{2}+\left(3 c^{2}+i \alpha\right) \log \left(-i \xi^{2}\right)+\phi_{0}+O\left(\xi^{-1}\right)$.

Proof. We need to rewrite (2.2) to be as follows:

$$
\begin{aligned}
W^{\prime 2}+W^{2}= & \frac{\sqrt{2}}{8} t^{-3 / 2} W+\frac{3 \sqrt{2}}{16} \int^{t} t^{-5 / 2} W d t-\frac{3}{16} t^{-2} W^{2}-\frac{3}{8} \int^{t} t^{-3} W^{2} d t \\
& -\frac{3 \sqrt{2}}{2} t^{-1 / 2} W^{3}-\frac{3 \sqrt{2}}{4} \int^{t} t^{-3 / 2} W^{3} d t-\frac{13}{8} t^{-1} W^{4}-\frac{13}{8} \int^{t} t^{-2} W^{4} d t \\
& -\frac{3 \sqrt{2}}{8} t^{-3 / 2} W^{5}-\frac{9 \sqrt{2}}{16} \int^{t} t^{-5 / 2} W^{5} d t-\frac{1}{16} t^{-2} W^{6}-\frac{1}{8} \int^{t} t^{-3} W^{6} d t \\
& +\frac{\hat{\alpha} \sqrt{2}}{2} t^{-1 / 2} W+\frac{\hat{\alpha} \sqrt{2}}{4} \int^{t} t^{-3 / 2} W d t+\frac{\hat{\alpha}}{4} t^{-1} W^{2}+\frac{\hat{\alpha}}{4} \int^{t} t^{-2} W^{2} d t \\
& -\frac{\beta}{8 t w^{2}}-\frac{\beta \sqrt{2}}{8} \int^{t} \frac{d t}{t^{2} w^{3}}+C .
\end{aligned}
$$

Then, $C=\lim _{t \rightarrow \infty}\left(W^{\prime 2}(t)+W^{2}(t)\right)$. If $C=0$, then $W \rightarrow 0$ as $t \rightarrow+\infty$. In this case, we can solve (2.1) to obtain that $W(t) \sim(\alpha \sqrt{2} / 4) t^{-1 / 2}$, and $W^{\prime}=O\left(t^{-3 / 2}\right)$. Thus, $w \sim \sqrt{2}+(\hat{\alpha} \sqrt{2} / 4) t^{-1}$ and $w^{\prime}=-(1 / 2) t^{-3 / 2} W+t^{-1 / 2} W^{\prime}=O\left(t^{-2}\right)$. If $C \neq 0$, we let

$$
W(t)=\rho(t) \cos \phi, \quad W^{\prime}(t)=\rho(t) \sin \phi .
$$


Then,

$$
\rho^{2}(t)=C+O\left(t^{-1 / 2}\right)
$$

Now, we let $C=c^{2}$ and find the asymptotics of $\phi$

$$
\begin{aligned}
\frac{d \phi}{d t} \\
=-1+\frac{W^{\prime \prime} W+W^{2}}{W^{\prime 2}+W^{2}} \\
=-1+\frac{-(9 \sqrt{2} / 4) t^{-1 / 2} W^{3}-(13 / 4) t^{-1} W^{4}+(\hat{\alpha} \sqrt{2} / 4) t^{-1 / 2} W+(\hat{\alpha} / 4) t^{-1} W^{2}+O\left(t^{-3 / 2}\right)}{c^{2}-(3 \sqrt{2} / 2) t^{-1 / 2} W^{3}+(\hat{\alpha} \sqrt{2} / 2) t^{-1 / 2} W+O\left(t^{-1}\right)} \\
=-1-\frac{9 \sqrt{2}}{4 c^{2}} t^{-1 / 2} W^{3}+\frac{\hat{\alpha} \sqrt{2}}{4 c^{2}} t^{-1 / 2} W-\frac{13}{4 c^{2}} t^{-1} W^{4}+\frac{\hat{\alpha}}{4 c^{2}} t^{-1} W^{2} \\
-\frac{27}{4 c^{4}} t^{-1} W^{6}+\frac{3 \hat{\alpha}}{c^{4}} t^{-1} W^{4}-\frac{\hat{\alpha}^{2}}{4 c^{4}} t^{-1} W^{2}+O\left(t^{-3 / 2}\right) .
\end{aligned}
$$

This implies that $W$ behaves like cost roughly. Thus, we need to pay attention to the terms with even power of $W$ which will give significant contribution to the expression of $\phi$ when we use integration by parts later. Using (2.12), we can find the asymptotic representation of $d t$ in terms of $d \phi$ as follows:

$$
d t=\left(1-\frac{9 \sqrt{2}}{4 c^{2}} t^{-1 / 2} W^{3}+\frac{\widehat{\alpha} \sqrt{2}}{4 c^{2}} t^{-1 / 2} W+O\left(t^{-1}\right)\right)(-d \phi) .
$$

Plugging (2.13) back to (2.12), we have

$$
\begin{aligned}
& d \phi=-d t+\left(-\frac{9 \sqrt{2}}{4 c^{2}} t^{-1 / 2} W^{3}+\frac{\hat{\alpha} \sqrt{2}}{4 c^{2}} t^{-1 / 2} W\right)\left(1-\frac{9 \sqrt{2}}{4 c^{2}} t^{-1 / 2} W^{3}\right. \\
& \left.+\frac{\hat{\alpha} \sqrt{2}}{4 c^{2}} t^{-1 / 2} W+O\left(t^{-1}\right)\right)(-d \phi) \\
& +\left(-\frac{13}{4 c^{2}} t^{-1} W^{4}+\frac{\hat{\alpha}}{4 c^{2}} t^{-1} W^{2}-\frac{27}{4 c^{4}} t^{-1} W^{6}+\frac{3 \hat{\alpha}}{c^{4}} t^{-1} W^{4}\right. \\
& \left.-\frac{\widehat{\alpha}^{2}}{4 c^{4}} t^{-1} W^{2}+O\left(t^{-3 / 2}\right)\right) d t \\
& =-d t+\left(-\frac{9 \sqrt{2}}{4 c^{2}} t^{-1 / 2} W^{3}+\frac{\hat{\alpha} \sqrt{2}}{4 c^{2}} t^{-1 / 2} W\right)(-d \phi) \\
& +\left(\frac{81}{8 c^{4}} t^{-1} W^{6}-\frac{9 \hat{\alpha}}{4 c^{4}} t^{-1} W^{4}+\frac{\hat{\alpha}^{2}}{8 c^{4}} t^{-1} W^{2}+O\left(t^{-3 / 2}\right)\right)(-d \phi) \\
& +\left(-\frac{13}{4 c^{2}} t^{-1} W^{4}+\frac{\hat{\alpha}}{4 c^{2}} t^{-1} W^{2}-\frac{27}{4 c^{4}} t^{-1} W^{6}+\frac{3 \hat{\alpha}}{c^{4}} t^{-1} W^{4}\right. \\
& \left.-\frac{\hat{\alpha}^{2}}{4 c^{4}} t^{-1} W^{2}+O\left(t^{-3 / 2}\right)\right) d t \text {. }
\end{aligned}
$$


By (2.9) and (2.10),

$$
\begin{aligned}
W & =\left(c-\frac{3 \sqrt{2}}{2} t^{-1 / 2} W^{3}+\frac{\hat{\alpha} \sqrt{2}}{2} t^{-1 / 2} W+O\left(t^{-1}\right)\right)^{1 / 2} \cos \phi \\
& =\left(c-\frac{3 \sqrt{2}}{4 c} t^{-1 / 2} W^{3}+\frac{\hat{\alpha} \sqrt{2}}{4 c} t^{-1 / 2} W+O\left(t^{-1}\right)\right) \cos \phi .
\end{aligned}
$$

Plugging (2.15) back to (2.14), we obtain

$$
\begin{aligned}
d \phi= & d t+\left(-\frac{9 c \sqrt{2}}{4} t^{-1 / 2} \cos ^{3} \phi+\frac{\hat{\alpha}}{4 c} t^{-1 / 2} \cos \phi\right)(-d \phi) \\
+ & \left(\frac{81}{8 c} t^{-1} W^{3} \cos ^{3} \phi-\frac{27 \hat{\alpha}}{8 c} t^{-1} W \cos ^{3} \phi-\frac{3 \hat{\alpha}}{8 c^{3}} t^{-1} W^{3} \cos \phi\right. \\
& \left.+\frac{\hat{\alpha}^{2}}{8 c^{3}} t^{-1} W \cos \phi\right)(-d \phi) \\
+ & \left(\frac{81}{8 c^{4}} t^{-1} W^{6}-\frac{9 \hat{\alpha}}{4 c^{4}} t^{-1} W^{4}+\frac{\hat{\alpha}^{2}}{8 c^{4}} t^{-1} W^{2}+O\left(t^{-3 / 2}\right)\right)(-d \phi) \\
+ & \left(-\frac{13}{4 c^{2}} t^{-1} W^{4}+\frac{\hat{\alpha}}{4 c^{2}} t^{-1} W^{2}-\frac{27}{4 c^{4}} t^{-1} W^{6}+\frac{3 \hat{\alpha}}{c^{4}} t^{-1} W^{4}\right. \\
& \left.-\frac{\hat{\alpha}^{2}}{4 c^{4}} t^{-1} W^{2}+O\left(t^{-3 / 2}\right)\right) d t \\
= & -d t+\left(-\frac{9 c \sqrt{2}}{4} t^{-1 / 2} \cos ^{3} \phi+\frac{\hat{\alpha}}{4 c} t^{-1 / 2} \cos \phi\right)(-d \phi) \\
+ & \left(-\frac{27 c^{2}}{2} t^{-1} \cos ^{6} \phi+\frac{13 c^{2}}{4} t^{-1} \cos ^{4} \phi+3 \hat{\alpha} t^{-1} \cos ^{4} \phi+O\left(t^{-3 / 2}\right)\right) d \phi \\
= & -d t+\left(3 c^{2}-\hat{\alpha}\right) t^{-1} d t \\
& +\left(-\frac{9 c \sqrt{2}}{4} t^{-1 / 2} \cos ^{3} \phi+\frac{\hat{\alpha}}{4 c} t^{-1 / 2} \cos ^{4} \phi+\frac{27 c^{2}}{2} t^{-1}\left(\cos ^{6} \phi-\frac{5}{16}\right)\right)(-d \phi) \\
& +\left(\left(\frac{13 c^{2}}{4}+3 \hat{\alpha}\right) t^{-1}\left(\cos ^{4} \phi-\frac{3}{8}\right)+\frac{\hat{\alpha}}{4} t^{-1}\left(\cos ^{2} \phi-\frac{1}{2}\right)+O\left(t^{-3 / 2}\right)\right) d \phi .
\end{aligned}
$$

Therefore, $\phi=-t+\left(3 c^{2}-\hat{\alpha}\right) \log t+\phi_{0}+O\left(t^{-1 / 2}\right)$.

Using transformations (1.4), (1.6), (2.10), and the one in Theorem 2.1, we get the asymptotic representation in the theorem and finish the proof of the theorem.

\section{REFERENCES}

[1] A. P. Bassom, P. A. Clarkson, C. K. Law, and J. B. McLeod, Application of uniform asymptotics to the second Painlevé transcendent, Arch. Rational Mech. Anal. 143 (1998), no. 3, 241-271.

[2] P. A. Clarkson and J. B. McLeod, Integral equations and connection formulae for the Painlevé equations, Painlevé Transcendents (Sainte-Adèle, PQ, 1990), NATO Adv. Sci. Inst. Ser. B Phys., vol. 278, Plenum, New York, 1992, pp. 1-31. 
[3] A. R. Its and V. Yu. Novokshenov, The Isomonodromic Deformation Method in the Theory of Painlevé Equations, Lecture Notes in Mathematics, vol. 1191, Springer-Verlag, Berlin, 1986.

[4] A. V. Kitaev, The method of isomonodromy deformations and the asymptotics of solutions of the "complete" third Painlevé equations, Math. USSR-Sb. 62 (1989), no. 2, 421-444.

[5] Y. Lu, Application of connection formula on eliminating plausible asymptotic representation of the Painlevé transcendents, submitted to Appl. Anal.

[6] _ _ An asymptotic representation of the general fourth Painleve transcendents, submitted to Int. J. Appl. Math.

[7] J. B. McLeod and C. B. Wang, General asymptotics for the Painlevé III transcendent, personal communication.

Youmin Lu: Department of Mathematics, Computer Science and Statistics, College of Science and Technology, Bloomsburg University of Pennsylvania, Bloomsburg, PA 17815, USA

E-mail address: y $1 \mathrm{u} @ \mathrm{~b} 1 \mathrm{oomu} . \mathrm{edu}$ 


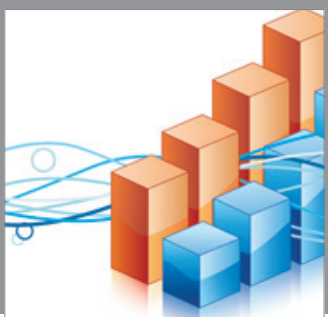

Advances in

Operations Research

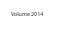

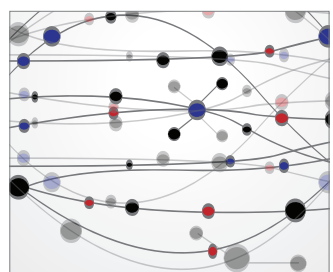

\section{The Scientific} World Journal
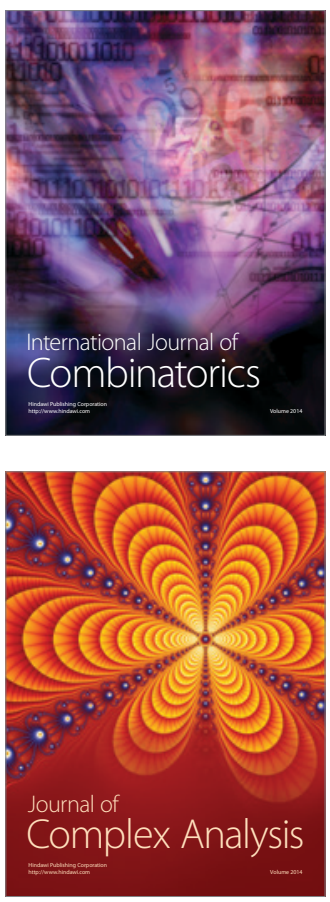

International Journal of

Mathematics and

Mathematical

Sciences
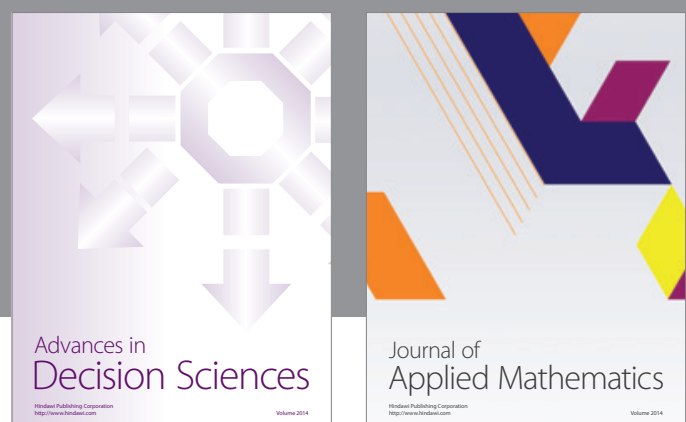

Journal of

Applied Mathematics
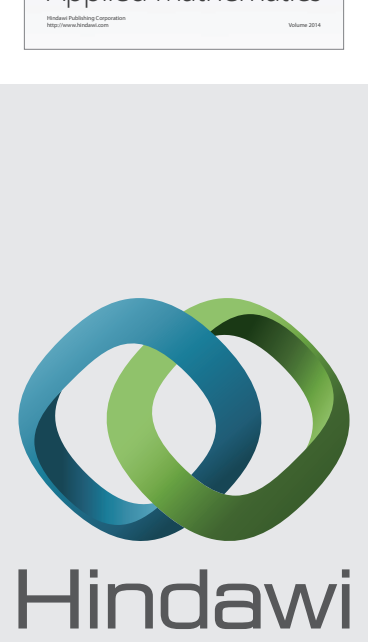

Submit your manuscripts at http://www.hindawi.com
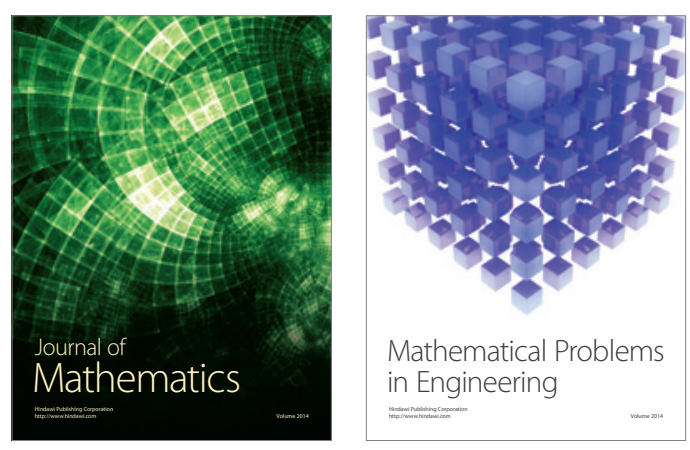

Mathematical Problems in Engineering
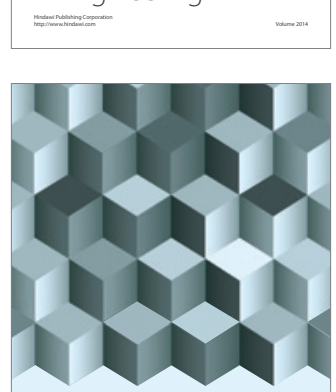

Journal of

Function Spaces
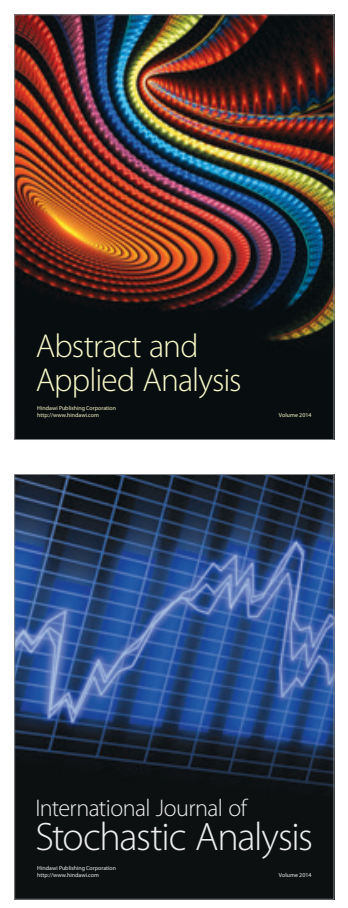

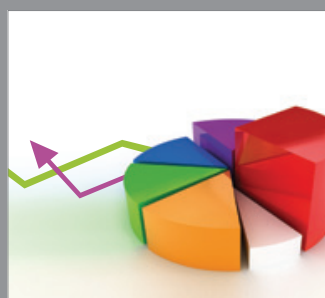

ournal of

Probability and Statistics

Promensencen
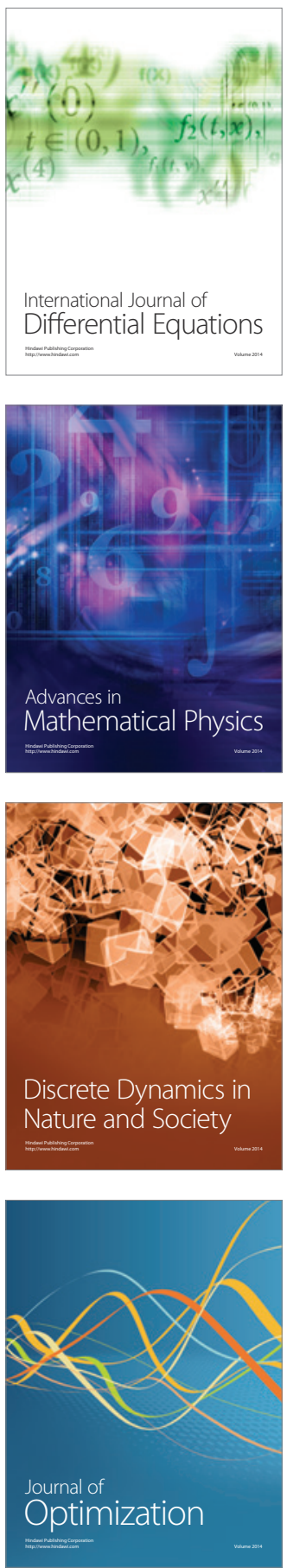